\title{
Molecular cloning and functional characterization of a mouse ccl6 analog gene in the rat
}

\author{
N. Jiang ${ }^{1}$, Y.H. Zheng ${ }^{2}$, X.J. Chen ${ }^{5}$, C. Qiu ${ }^{4}$, X.F. Zhang ${ }^{4}$, S.H. Wen ${ }^{4}$ and \\ G.X. Bian $^{3,4,5}$ \\ ${ }^{1}$ Department of Pharmacy, Beijing Military Region, \\ General Hospital, Beijing, China \\ ${ }^{2}$ Department of Forensic Science, School of Basic Medical, \\ Southern Medical University, Guangzhou, China \\ ${ }^{3}$ State Key Laboratory of Proteomics, Beijing, China \\ ${ }^{4}$ Beijing Proteome Research Center, Beijing, China \\ ${ }^{5}$ Beijing Institute of Radiation Medicine, Beijing, China \\ Corresponding author: N. Jiang / G.X. Bian \\ E-mail: jiangnan7757@sohu.com / bian2222@yahoo.com
}

Genet. Mol. Res. 11 (4): 3889-3898 (2012)

Received March 12, 2012

Accepted August 8, 2012

Published November 12, 2012

DOI http://dx.doi.org/10.4238/2012.November.12.6

\begin{abstract}
Suppression subtractive hybridization was used to analyze differential expression of genes in rat peritoneal macrophages after granulocyte macrophage colony-stimulating factor treatment. We identified and cloned the mouse $\mathrm{C} 10$ analog gene in the rat, and named it as ccl6. The full-length cDNA of rat ccl6 was $467 \mathrm{bp}$, which contains a single-open reading frame and encodes 116 amino acid residues. Compared with other $\mathrm{C}-\mathrm{C}$ chemokines, the rat ccl6 gene had an unusual four-exon genome structure instead of the typical three exons, it had the highest homology with murine ccl6. The rat ccl6 gene was localized on chromosome 10, where most of the C-C chemokine superfamily members are located. The recombinant rat $\mathrm{C}-\mathrm{C}$ chemokine ligand 6 (CCL6) protein was expressed by the pGEX4T-1 plasmid in Escherichia coli BL21. The purified recombinant protein had bioactivity similar to that of mouse CCL6, which is a chemoattractant for macrophages and
\end{abstract}


lymphocytes, but not for neutrophils.

Key words: $c c l 6$; Genome structure; Chemoattractant; Macrophages; Lymphocytes

\section{INTRODUCTION}

C-C chemokine ligand 6 (CCL6, or C10) is a C-C chemokine that has been cloned from mouse (Berger et al., 1993). CCL6 is typically produced by activated macrophages, lymphocytes, and fibroblasts, and is stimulated by exposure to interleukin (IL)-3, IL-4, IL-13, and granulocyte macrophage colony-stimulating factor (GM-CSF; Orlofsky et al., 1994). This novel chemokine shares high homology with human $\mathrm{C}-\mathrm{C}$ chemokine, macrophage inflammatory protein-related protein 1 (MRP-1). Both of these chemokines are chemotactic for monocytes and T cells. Previous studies demonstrated that CCL6 had multiple activities (Olson and Ley, 2002). Furthermore, CCL6 is differentially regulated by Thelper (Th) 1 and Th2 cytokines (Orlofsky et al., 2000), and is also involved in a variety of chronic inflammatory disorders (Belperio et al., 2002).

Although mouse CCL6 has been extensively studied, its human or rat homologue is still unknown. In our previous study, we used the suppression subtractive hybridization (SSH) method to analyze the differential expression of genes in the rat peritoneal macrophages after stimulation with GM-CSF. Sequencing results showed that one positive clone, SSH-8, was the homologue of mouse $c c l 6$. In this study, we cloned the homologue of ccl6 from GM-CSFtreated rat macrophages. The novel chemokine was expressed in a prokaryotic system and purified. The purified protein had chemotactic effects on rat lymphocytes and macrophages, but not on neutrophils in vitro, which are similar to the effects of mouse CCL6 on these cells. These findings would be useful in the mechanistic study of inflammatory disorders using rat models.

\section{MATERIAL AND METHODS}

\section{Database search and full-length cDNA deduction}

SSH-8-related sequences were searched in dbEST (http://www.ncbi.nlm.nih.gov/ $\mathrm{dbEST} /$ ) using the default parameters of the BLASTN program (http://www.ncbi.nlm.nih. gov/blast). A number of rat expressed sequence tags (ESTs) were identified using a method described elsewhere (Lou et al., 2003). These ESTs were retrieved and a contig was assembled from overlapping ESTs using the EST Assembly machine on the World Wide Web (http://www.tigen.it). The accession numbers of ESTs used for rat ccl6 analog assembly were AW917998, AA848632, AI169984, AA800480, AI234153, BF555934, and BE095824.

\section{Cell culture and cloning of rat ccl6}

Rat macrophages were purified from the parietal peritoneum and cultured in Dulbecco's modified Eagle's medium (supplemented with $100 \mathrm{U} / \mathrm{mL}$ ampicillin and $100 \mu \mathrm{g} /$ $\mathrm{mL}$ streptomycin). Cultured cells were treated with $50 \mathrm{ng} / \mathrm{mL}$ GM-CSF for $24 \mathrm{~h}$, and total RNA was extracted from cells using Trizol (lot: 1148608, Invitrogen, Carlsbad, USA), according to manufacturer instructions. For reverse transcriptase (RT)-PCR, total RNA was 
reverse transcribed using $600 \mathrm{U}$ Moloney murine leukemia virus reverse transcriptase and oligo (dT; Takara) at $37^{\circ} \mathrm{C}$ for 60 min. Nested PCR was performed using the following primers: P1: 5'-AGACCAGCTGGGCCTGTCCT-3'; P2: 5'-ATGTCAGGCTGAATCCATGTGAA-3'; P3: 5'-CAGGATGATGAGACATTCC-3'; P4: 5'-GGAGACAGAAGAATTCAGG-3'. After gel extraction, the PCR product was inserted into the pMD18-T vector (Takara) and named pMD18-T/ccl6. The accuracy of pMD18-T/ccl6 was confirmed by enzyme digestion and sequencing analysis by Genecore Corp. (Shanghai). The characteristics of the cDNA fragments and their putative amino acid sequences were analyzed using the DNAsis software (HITACHI).

\section{Recombinant rat CCL6 production and purification}

The coding region of rat $c c l 6$ was amplified from the vector pMD18-T/ccl6 using primers P3 (5'-ATGCGGATCCATGATGAGACATTCCAAGAC-3') and P4 (5'-ATGCGA ATTCAAGCAATGGCACTG-3'), in which a BamHI site was inserted at the 5'-region and an EcoRI site was inserted at the 3'-region. The PCR product and pGEX4T-1 (Pharmacia) expression vector were digested with BamHI and EcoRI. After extraction from gels, the ccl6 fragment was inserted into the vector. The recombinant plasmid was pGEX4T-1/ccl6, and the accuracy of insertion was verified by restriction enzyme digestion.

The pGEX4T-1/ccl6 vector was transformed into Escherichia coli (BL21 strain) by a standard protocol. The recombinant protein was linked to glutathione S-transferase (GST), named GST-CCL6 protein. The GST-CCL6 protein was extracted from bacterial cells by sonication in phosphate-buffered saline (PBS) containing 1\% Triton X-100 and purified by glutathione-Sepharose 4B column (Pharmarcia; Ikadai et al., 1999).

The purified fusion protein was cleaved with thrombin $(10 \mathrm{U} / \mathrm{mg}$ fusion protein, Amersham Pharmacia Biotech) overnight (12 h) at room temperature, and then eluted by PBS (Williamson et al., 2000). The solution was purified by glutathione-Sepharose $4 \mathrm{~B}$ column again to remove the fusion GST tag. The solution was loaded onto a Sephacryl-G75 column $(2.6 \times 80$ $\mathrm{cm}$ ) to obtain a purified CCL6 protein. Protein samples were analyzed by $15 \%$ polyacrylamide gel using sodium dodecyl sulfate-polyacrylamide gel electrophoresis (SDS-PAGE).

\section{Chemotaxis assay}

Purified neutrophils were resuspended at $1 \times 10^{6}$ cells $/ \mathrm{mL}$ in Hank's balanced salt solution (HBSS), and macrophages and lymphocytes were resuspended at $2 \times 10^{6}$ cells $/ \mathrm{mL}$ and $5 \times 10^{6} \mathrm{cells} / \mathrm{mL}$ in Roswell Park Memorial Institute medium (RPMI) 1640 (Life Technologies) supplemented with $0.5 \%$ low endotoxin bovine serum albumin (BSA) and $20 \mathrm{mM}$ 4-(2-hydroxyethyl)-1-piperazineethanesulfonic acid (HEPES), respectively. Cell migration was measured using a 48-well chemotaxis chamber as previously reported (Sarafi et al., 1997). The lower wells contained the purified rat CCL6 protein, and the medium without the purified rat CCL6 protein was set as the untreated control. The pore size of the polycarbonate membrane (Millipore) for the neutrophils was $3 \mu \mathrm{m}$ and the incubation time was $30 \mathrm{~min}$. For monocytes and lymphocytes, a $5-\mu \mathrm{m}$ pore membrane was used and the incubation time was 90 $\mathrm{min}$. The cells that passed through the pore of membrane were fixed and stained. Stained cells were counted in 3 random fields (400X magnification) under light microscope. Results were represented as means \pm standard deviation from triplicate samples. 


\section{RESULTS}

\section{SSH screen and the deduced sequence for rat ccl6}

Using the SSH method, we found that 26 positive clones were homologous to mouse ccl6. The searching sequences from the GenBank database revealed that the fragment of SSH-8 might be a novel gene. Using this sequence to search the EST database, a number of ESTs were identified. With help of the EST assembly machine, we spliced a full-length rat gene (Figure 1). It included a single polyadenylation signal AATAAA and a poly(A) tail. Based on rat genome information, it was located on chromosome 10, where most of the rat chemokines are located.

$\begin{array}{lr}\text { GATCCCCAGACCAGCTGGGCCTGTCCTTCAGGATGATGAGACATTCCAAGACTGCCATT } & 60 \\ \text { TCATTCTTATCCTTGTGGCCTCCTTGGGTCCAGGCTGGGCTCATACAAGATACGGTA } & 120 \\ \text { AAAGAAGATCGTCCCTTAATCCTACAATAATCACCAAGGCTTTCAAGACTCTTCAGAC } & 180 \\ \text { TGCTGCTTCTCCTATGCCTCACAGATCCCATGTTCAAGATTTATATATTATTTCCAAC } & 240 \\ \text { CAGTGGTGGGGCACCAAGCCAGGCATCATCTTTGTCACCAGGAAGAGGAAGCGGGTCTG } & 300 \\ \text { TGCCAACCCGAGTGATCAGAGAGTTCAGACGTGCATAAGCACCCTGAAGCTAGGCCCAAG } & 360 \\ \text { ATCTGGGAAAGTGCCATTGCTTGAGAAGGAGGGCGTACTGCCACCTGCATTCTTCTGTC } & 420 \\ \text { TCCCCAGTGACCACCTAGGAAGCGCTAAGCATTTGTTTTATAGATATTTAAAGCACGTA } & 480 \\ \text { TTCTTCTGTACAGGTTTAAAGCAGCACATGTAGTCCACAGTATTCACATGGATTCAGCCT } & 540 \\ \text { GACATGATTAGAGCCATCTCGAGGTGTAACCATCATGAGTGTCTTTGGGTAACTGTTGGG } & 600 \\ \text { TTTTCTTGCATAGTTCCTCAGCAGATTATAAATGGATAAACTACTAGTCTTTGGGACTTT } & 660 \\ \text { GGAATTGTCTGGTTTGATGCAAGCTTAAGCCAGGTGTATCCAGCTGAGATGAAATCAA } & 720 \\ \text { TTTTTGCCCTAAGCCATACATATGTCCAACTTTGTGGGGACCCAGCTGTTCTTCCTGCCA } & 780 \\ \text { CCATAGAGCAGTAAGTGACCCCAATAAAGTGCACTCCATGTAAAAAAAAAAAAAAAAA } & 838\end{array}$

Figure 1. Complete assembled contig sequence of ESTs. The letters in bold are the encoding region.

\section{Cloning and sequencing of rat $\mathrm{ccl} 6$}

We used the nested primers to localize the upstream start codon and the downstream stop codon, and the full-length cDNA of rat $c c l 6$ was successfully obtained from GM-CSF-stimulated rat macrophages by RT-PCR. The cDNA fragment was successfully cloned into the pMD18-T vector and checked by sequencing. It was demonstrated to be a novel gene by the sequencing results. The full-length cDNA was uploaded to GenBank (accession No. AY620903). The predicted amino acid sequence and NCBI Conserved Domain Search results are shown in Figure 2. There was only 1 open reading frame with an ATG initiation codon at nucleotide 26. It encoded a polypeptide of 116 total residues with a calculated molecular mass of $12.8 \mathrm{kDa}$. The peptide sequence had a typical C-C chemokine motif compared to Conserved Domain Database at GenBank (http://www.ncbi.nlm.nih. gov/sites/entrez?db=cdd; Petrenko et al., 1995). We compared it with mouse C-C chemokines using CLUSTALW (http://www.biosino.org/pages/lab.htm; Figure 3A). It shared 79\% sequence homol- 
ogy with mouse CCL6 (Figure 3B). The deduced rat CCL6 protein did not contain the putative glycosylation site and typical signal cleavage site, based on the searching on the Signal P server (Nielsen et al., 1999); thus, we chose the prokaryotic system to express it.

$\begin{array}{|rrrrrr|}10 & 20 & 30 & 40 & 50 & 60 \\ \text { HIMRHSKTAIS } & \text { FFILVAVLGS } & \text { QAGLIQDTVK EDRPFNPTII } & \text { HQGFQDSSDC } & \text { CFSYASQIPC } \\ 70 & 80 & 90 & 100 & 110 & 120 \\ \text { SRFIYYFPTS } & \text { GGCTKPGIIF } & \text { VIRKRKRVCA } & \text { NPSDQRVOTC } & \text { ISTLKLGPRS } & \text { GNSAIA.... }\end{array}$

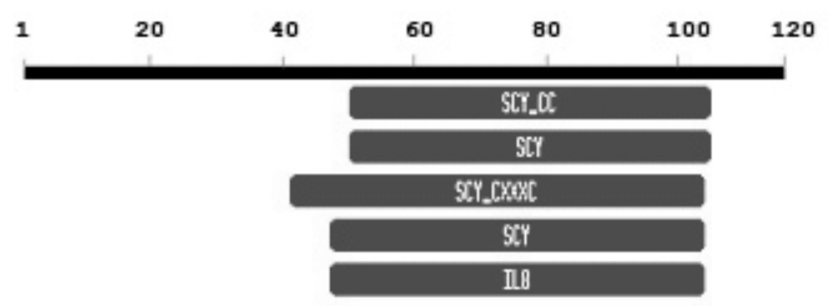

Figure 2. Predicted amino acid sequence and NCBI Conserved Domain Search result.

A

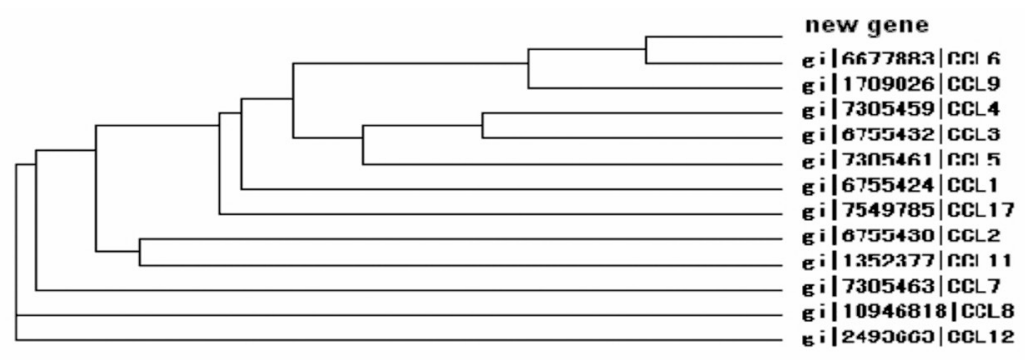

B

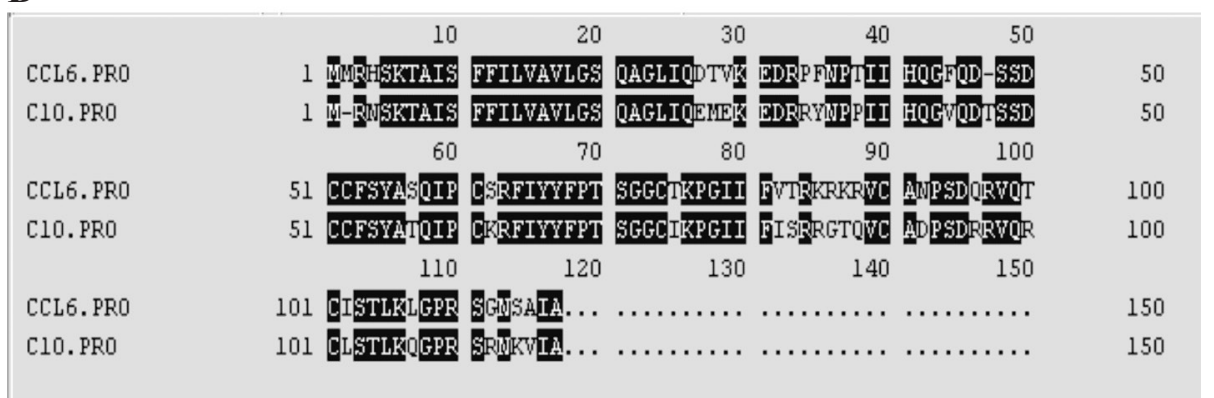

Figure 3. Alignment of the new gene with other chemokines. A. Alignment of the new gene with other mouse C-C chemokines using CLUSTALW. B. Alignment of the new gene with mouse CCL6; rat CCL6 shares 79\% homology with mouse CCL6. Conserved amino acids are shown in black background. 
We also revealed that the new gene sequences were similar to 4-interval fragments in the chromosome. The mouse ccl6 gene has 4 exons, and exons 3 and 4 of ccl6 contain 4 cysteines in the same manner as those in exons 2 and 3 of other beta-chemokine family members. In this study, we observed that the structure of rat ccl6 was very similar to that of mouse $c c l 6$ (Figure 4A and B).

\section{A}

Exon 1

\section{CTC ATA CAA}

Exon2

ATT CAC CAA

Exon3

CAG GCA TCA

B

Chromosome 10

Rat CCL6

Mouse CCL6

C-C Chemokine
Intron 1

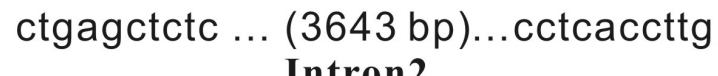

Intron 2

taaacgacca...(385 bp) ...ctcttcctac

Intron 3

gtaagtgtc ...(332 bp) ...tttctcttc
Exon2

AGATAC GGT

Exon3

GCT TTC AAG

Exon 4

ATC TTT GTC
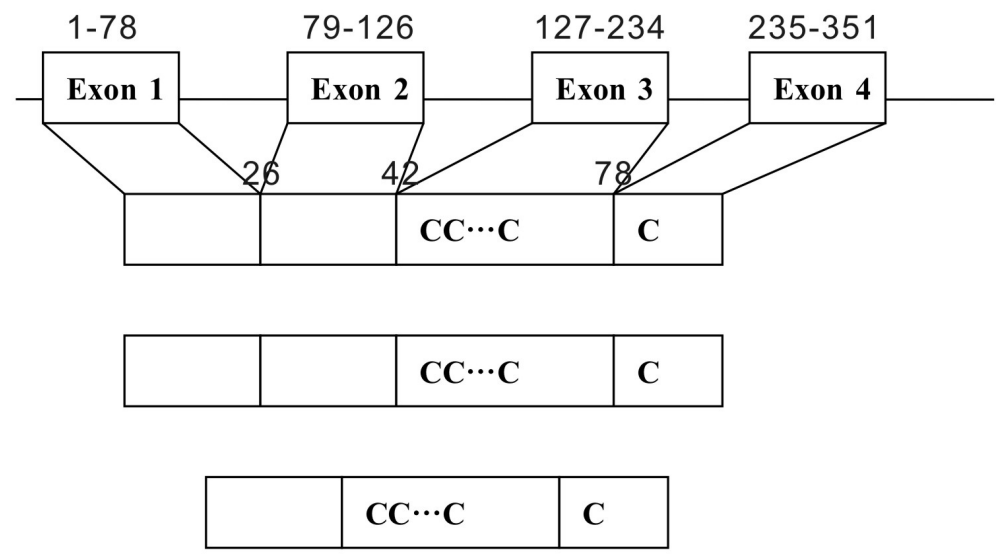

Figure 4. A. Sequences of the intron-exon junctions in rat ccl6. Exons are shown in capital letters; intron sequences are shown in lower case letters. The length of each intron is shown in parentheses. B. Sketch map of rat CCL6, mouse CCL6 and other C-C chemokines. Rat CCL6 and mouse CCL6 have four exons (the third exon contains the first three of four conserved cysteines, and the fourth exon contains the last conserved cysteine), while most other C-C chemokines only have three exons.

\section{Expression and purification of rat CCL6}

The recombinant rat CCL6 protein was expressed in a soluble form and obtained as a fusion protein with GST. The protein had an apparent molecular size of $39 \mathrm{kDa}$, which was equal to the predicted molecular weight of the fusion protein (Figure 5). The recombinant fusion protein constituted approximately $15 \%$ of total cellular proteins in the bacterial cells. The purification procedure for rat CCL6 polypeptide production is shown in Figure 6. The final optimized yield of rat CCL6 was approximately 3-4 mg/L culture broth, which was higher than the typical yield of $2.5 \mathrm{mg} / \mathrm{L}$ for GST fusion proteins (Amersham Pharmacia Biotech). 


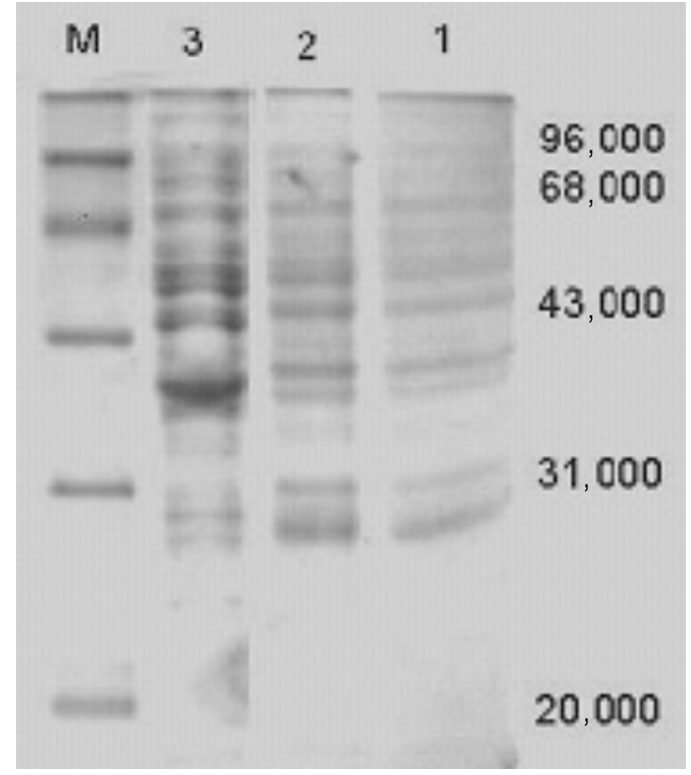

Figure 5. SDS-PAGE analysis for total proteins from Escherichia coli bacterial cells: lane $1=$ crude lysate of BL21 cells with IPTG induction; lane 2 = crude lysate of BL21 cells that are transformed with pGEX4T-1 and induced by IPTG; lane 3 = crude lysate of BL21 cells that are transformed with GEX-4T-1/ccl6 and induced by IPTG; lane $M=$ molecular weight marker.

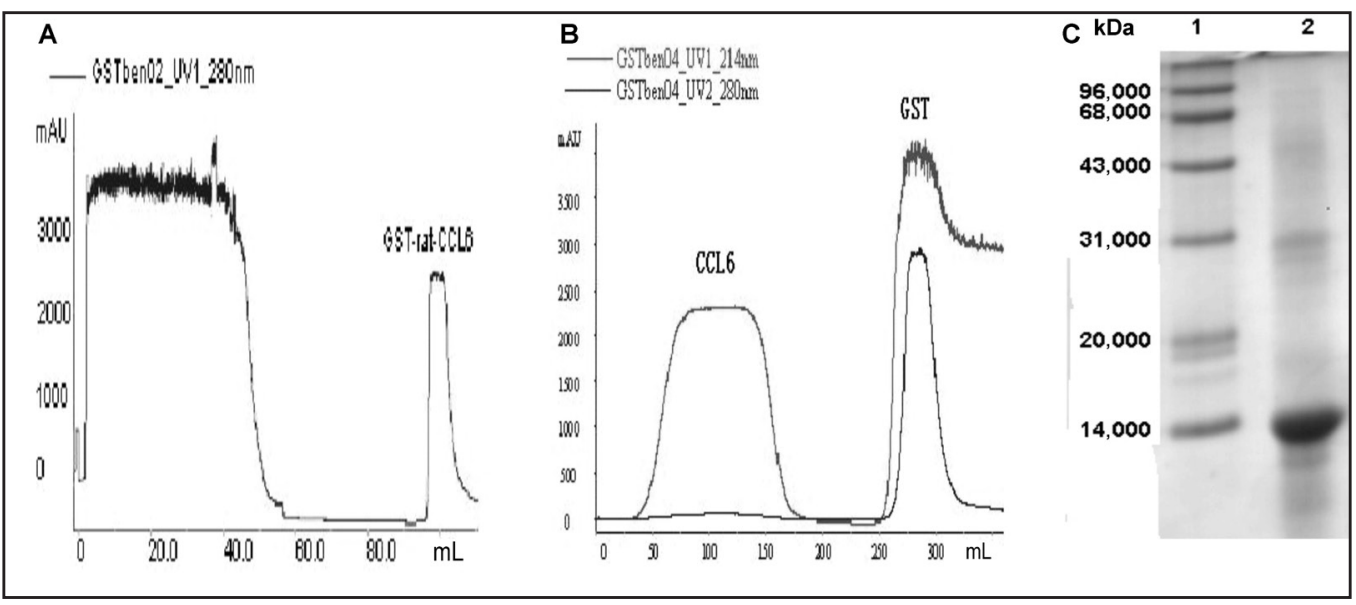

Figure 6. Purification of CCL6 protein. A. Glutathione Sepharose 4B affinity column to purify fusion protein. B. Gel filtration pattern on G75 of eluent from Glutathione Sepharose 4B after thrombin digestion. C. SDS-PAGE analysis for purified rat CCL6. Lane $1=$ molecular weight marker; lane $2=$ purified rat CCL6. GST = glutathione S-transferase.

\section{Chemotactic activities of recombinant rat CCL6}

To confirm the chemotactic activity of rat CCL6, the effect of rat CCL6 on the migra- 
tion of macrophages and lymphocytes was examined using a chemotaxis chamber (Aarvak et al., 1999). The chemotactic activity results were represented by the percentage obtained by the number of migrated cells in the medium containing rat CCL6 divided by the number of migrated cells in the control medium without rat CCL6. Compared with the control medium, the medium containing rat CCL6 induced an obvious chemotactic response in rat macrophages and lymphocytes that peaked at $10 \mathrm{ng} / \mathrm{mL}$, although the response was reduced at higher concentrations in a bell-shaped chemotactic response curve (Han et al., 2001b). Rat CCL6 had no obvious effect on the migration of neutrophils in this experiment (Figure 7).

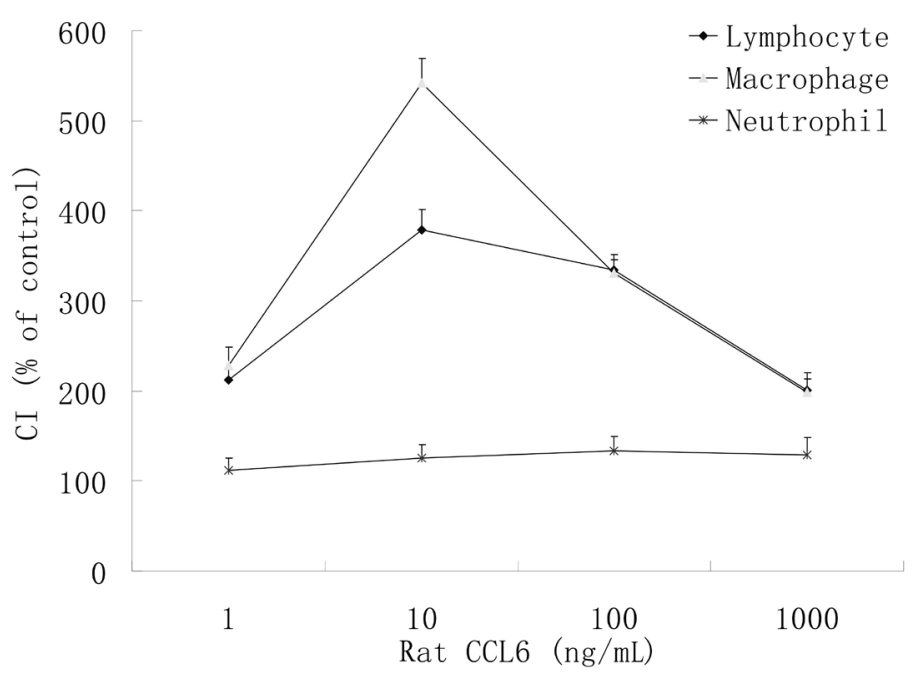

Figure 7. Chemotaxis of leukocyte subsets by rat CCL6. Leukocyte subsets were exposed to rat CCL6 (1 1000 $\mathrm{ng} / \mathrm{mL})$ and cell migration was determined. The medium without rat CCL6 was set as the untreated control. Values are means \pm standard deviation from three independent experiments. $\mathrm{CI}=$ (number of migrated cells in the medium with rat CCL6 $\div$ number of migrated cells in the control medium) x $100 \%$.

\section{DISCUSSION}

Chemokines comprise a broad family of chemoattractant cytokines that play important roles in several physiological and pathological processes (Li et al., 2000; Gerard and Rollins, 2001). Based on the appearance of 4 conserved cysteine residues, 4 subfamilies are distinguished. Chemokines are thought to have evolved from 1 ancestral gene, thus their sequences share high homology. Chemokines belong to a single subfamily and are clustered in 1 chromosome. Hundreds of chemokine genes have been reported, and they are highly conserved among humans, mice, and rats.

Herein we were interested in determining the mouse $c c l 6$ analog gene in rat. Although all initial attempts were unsuccessful to clone the mouse $c c l 6$ analog gene from human cDNA libraries, the search for its rat homologue was successfully conducted. In this study, SSH, a comprehensive and large-scale gene expression-based approach, was used to unbiasedly discover the genes that are induced or suppressed by a particular treatment. Furthermore, SSH allows one to identify differentially expressed cDNAs without prior knowledge of their sequence. The SSH-8 clone that contains the typical chemokine characteristic sequence was 
identified as a new sequence after comparing it with all known chemokine sequences. A number of rat ESTs were identified after searching this sequence in the EST database.

Most members of the $\mathrm{C}-\mathrm{C}$ chemokine subfamily have 3 exons, and the second exon contains the first 3 of the 4 conserved cysteines, and the third exon contains the last (Han et al., 2001a). In this study, we found that the rat $c c l 6$ gene contained 4 exons that are similar to the mouse $c c l 6$ gene. Thus, we named the new gene rat $c c l 6$.

Chemokine C10, also called MRP-1 or CCL6, is a C-C chemokine that was first isolated in 1991 from mouse bone marrow (Orlofsky et al., 1991). It is a macrophage chemoattractant both in vitro and in vivo. It is upregulated in experimental allergic bronchopulmonary aspergillosis during chronic peritoneal inflammation (Steinhauser et al., 2000), and in mouse models of cutaneous wound repair (Kaesler et al., 2002). Our chemotactic assays suggested that rat CCL6 was a major chemoattractant for macrophages and lymphocytes but not for neutrophils, which is similar to the chemotactic function of mouse CCL6. Mouse CCL6 can be selectively induced by IL-10 and GM-CSF, thus we cloned our new gene from GM-CSF-treated macrophages. The assembled ESTs are primarily derived from liver, kidney, heart, brain, and eye, which indicates that CCL6 may be expressed in these tissues. In addition, GM-CSF is probably an important inducer for rat CCL6.

In summary, we cloned the novel rat cytokine gene $c c l 6$ by the SSH method. Furthermore we showed that rat CCL6 and mouse CCL6 had similar characteristics and bioactivity.

\section{ACKNOWLEDGMENTS}

Research supported by the National Natural Science Foundation of China (\#32970432).

\section{REFERENCES}

Aarvak T, Chabaud M, Kallberg E, Miossec P, et al. (1999). Change in the Th1/Th2 phenotype of memory T-cell clones from rheumatoid arthritis synovium. Scand. J. Immunol. 50: 1-9.

Belperio JA, Dy M, Burdick MD, Xue YY, et al. (2002). Interaction of IL-13 and C10 in the pathogenesis of bleomycininduced pulmonary fibrosis. Am. J. Respir. Cell Mol. Biol. 27: 419-427.

Berger MS, Kozak CA, Gabriel A and Prystowsky MB (1993). The gene for C10, a member of the beta-chemokine family, is located on mouse chromosome 11 and contains a novel second exon not found in other chemokines. DNA Cell Biol. 12: 839-847.

Gerard C and Rollins BJ (2001). Chemokines and disease. Nat. Immunol. 2: 108-115.

Han W, Lou Y, Tang J, Zhang Y, et al. (2001a). Molecular cloning and characterization of chemokine-like factor 1 (CKLF1), a novel human cytokine with unique structure and potential chemotactic activity. Biochem. J. 357: 127-135.

Han WL, Rui M, Zhang YM, Chen YY, et al. (2001b). Stimulatory effect of chemokine-like factor 1 (CKLF1) on the growth of bone marrow cells. Zhongguo Yi Хue Ke Xue Yuan Хие Bao 23: 119-122.

Ikadai H, Xuan X, Igarashi I, Tanaka S, et al. (1999). Cloning and expression of a 48-kilodalton Babesia caballi merozoite rhoptry protein and potential use of the recombinant antigen in an enzyme-linked immunosorbent assay. J. Clin. Microbiol. 37: 3475-3480.

Kaesler S, Regenbogen J, Durka S, Goppelt A, et al. (2002). The healing skin wound: a novel site of action of the chemokine C10. Cytokine 17: 157-163.

Li QJ, Lu S, Ye RD and Martins-Green M (2000). Isolation and characterization of a new chemokine receptor gene, the putative chicken CXCR1. Gene 257: 307-317.

Lou Y, Xia D, Han W, Wang Y, et al. (2003). Molecular cloning and characterization of rat chemokine-like factor 1 and 2. Gene 307: 125-132.

Nielsen H, Brunak S and von Heijne G (1999). Machine learning approaches for the prediction of signal peptides and other protein sorting signals. Protein Eng. 12: 3-9.

Olson TS and Ley K (2002). Chemokines and chemokine receptors in leukocyte trafficking. Am. J. Physiol. Regul. Integr. 
Comp Physiol. 283: R7-28.

Orlofsky A, Berger MS and Prystowsky MB (1991). Novel expression pattern of a new member of the MIP-1 family of cytokine-like genes. Cell Regul. 2: 403-412.

Orlofsky A, Lin EY and Prystowsky MB (1994). Selective induction of the beta chemokine C10 by IL-4 in mouse macrophages. J. Immunol. 152: 5084-5091.

Orlofsky A, Wu Y and Prystowsky MB (2000). Divergent regulation of the murine CC chemokine C10 by Th(1) and $\operatorname{Th}(2)$ cytokines. Cytokine 12: 220-228.

Petrenko O, Ischenko I and Enrietto PJ (1995). Isolation of a cDNA encoding a novel chicken chemokine homologous to mammalian macrophage inflammatory protein-1 beta. Gene 160: 305-306.

Sarafi MN, Garcia-Zepeda EA, MacLean JA, Charo IF, et al. (1997). Murine monocyte chemoattractant protein (MCP)-5: a novel CC chemokine that is a structural and functional homologue of human MCP-1. J. Exp. Med. 185: 99-109.

Steinhauser ML, Hogaboam CM, Matsukawa A, Lukacs NW, et al. (2000). Chemokine C10 promotes disease resolution and survival in an experimental model of bacterial sepsis. Infect. Immun. 68: 6108-6114.

Williamson PT, Roth JF, Haddingham T and Watts A (2000). Expression and purification of recombinant neurotensin in Escherichia coli. Protein Expr. Purif. 19: 271-275. 